\title{
Role of tumor necrosis factor receptor-associated factor 6 in pyroptosis during acute pancreatitis
}

\author{
BIWEI WEI ${ }^{1 *}$, YAHUI GONG ${ }^{1,2^{*}}$, HUIYING YANG ${ }^{1}$, JIE ZHOU $^{1}$, ZHOU SU $^{1}$ and ZHIHAI LIANG ${ }^{1}$ \\ ${ }^{1}$ Department of Gastroenterology, The First Affiliated Hospital of Guangxi Medical University, Nanning, \\ Guangxi Zhuang Autonomous Region 530021; ${ }^{2}$ Department of Pediatrics, \\ Jingzhou Central Hospital, Jingzhou, Hubei 434020, P.R. China
}

Received June 2, 2021; Accepted September 17, 2021

DOI: $10.3892 / \mathrm{mmr} .2021 .12488$

\begin{abstract}
Acute pancreatitis (AP) is hypothesized to be related to the activation of an inflammatory response induced by pyroptosis. The aim of the present study was to investigate the potential role of tumor necrosis factor receptor-associated factor 6 (TRAF6) in pyroptosis in an AP rat model and the human pancreatic ductal epithelial HPDE6C7 cell line. In vivo, AP was induced by intraperitoneal injection of caerulein (CAE) in rats. The rats were sacrificed at 24 or $48 \mathrm{~h}$ after the final CAE injection. In vitro, HPDE6C 7 cells were treated with CAE for 12, 24 and $48 \mathrm{~h}$. Moreover, TRAF6 was overexpressed and treated with CAE for $48 \mathrm{~h}$. Histopathological changes of pancreatic, serum and supernatant inflammatory cytokines and pyroptosis-related mRNA and protein expression levels were determined by histopathological scores, ELISA, reverse transcription-quantitative PCR and western blotting. In addition, pyroptosis morphological changes were also determined by Hoechst/PI staining in HPDE6C7 cells. Results showed that AP was observed in the CAE-induced rat model, and that serum IL-1 $\beta$ and IL-18 levels, and TRAF6, NLR pyrin domain containing 3 (NLRP3), caspase-1 and caspase-3 mRNA and protein expression levels were increased. Similar in HPDE6C7 cells, CAE treatment caused supernatant IL-1 $\beta$ level, NLRP3 and caspase-1 mRNA expression levels to significantly increase. After TRAF6 overexpression and CAE treatment, supernatant IL-1 $\beta$ level, caspase-1 protein expression level, and NLRP3 and caspase-3 mRNA and protein expression levels were also significantly increased. Furthermore, cells exhibited red fluorescence in Hoechst/PI staining, which can
\end{abstract}

Correspondence to: Dr Zhihai Liang, Department of Gastroenterology, The First Affiliated Hospital of Guangxi Medical University, 6 Shuangyong Road, Nanning, Guangxi Zhuang Autonomous Region 530021, P.R. China

E-mail: ahhai@163.com

*Contributed equally

Key words: acute pancreatitis, pyroptosis, tumor necrosis factor receptor-associated factor 6 , caspase-1, caspase-3 be used as a method of detecting pyroptosis activation. The results also showed that the red fluorescence was stronger after CAE treatment or TRAF6 overexpression plus CAE treatment. In conclusion, TRAF6 and caspase-1/3 signaling pathways were involved in the pathogenesis of CAE-induced AP in rats. Pyroptosis was activated by CAE and TRAF6 overexpression via the caspase-1/3 signaling pathways in HPDE6C7 cells.

\section{Introduction}

Acute pancreatitis (AP) is a common disease, which can be classified as mild, moderate and severe. Severe disease develops in $20 \%$ of patients with AP, which progresses rapidly and can cause systemic inflammatory response syndrome and multiple organ dysfunction, leading to increased mortality (1). The pathogenesis of AP is not yet fully understood. Cell death caused by pyroptosis, necrosis, apoptosis and necroptosis, is involved in the pathogenesis of AP. Pyroptosis, apoptosis and necroptosis represent three pathways of genetically encoded necrotic cell death (2). Apoptosis is a highly regulated form of cell death, characterized by the activation of multiple caspases (3), and necroptosis is a form of regulated necrotic cell death mediated by receptor-interacting protein kinase (RIP) 1 and RIP3 (4,5). Unlike these, pyroptosis is a programmed cell death pathway, also known as caspase-1-mediated cell death, that induces the release of active IL-1 $\beta$ and IL-18 (2). Increasing evidence indicates a close relationship between AP and pyroptosis (6). However, the underlying mechanism by which pyroptosis induces pancreatic injury leading to AP has not been fully elucidated.

Pyroptosis is one of the main mechanisms of cell death and causes an acute inflammatory response by activating inflammasomes and releasing inflammatory cytokines (7). Previous studies have demonstrated that pyroptosis is involved in the occurrence and development of infectious diseases, cardiovascular diseases, cancer and numerous other diseases (8-12). Emerging studies suggest that toll-like receptors (TLRs) can be recognized as upstream signals by nucleotide-binding and oligomerization domain-like receptors (NLRs), which activate the assembly of the NLR pyrin domain containing 3 (NLRP3) inflammasome and caspase-1 and subsequently trigger pyroptosis (13-15). Tumor necrosis factor receptor-associated factor (TRAF)6 is a 
member of the TRAF protein family that serves a vital role in TLR signaling pathways $(16,17)$. Furthermore, TRAF6 is required for apoptosis-associated speck-like protein (ASC) oligomerization and the assembly of the NLRP3 inflammasome; TRAF6 deficiency specifically inhibits TLR/IL-1R-initiated NLRP3 inflammasome activation, caspase-1 cleavage and pyroptosis (18). At present, the roles of TRAF6 in AP and pyroptosis during AP are still unclear. Therefore, the present study aimed to determine the role of TRAF6 in rat and cell models of caerulein (CAE)-induced pancreatic injury. Pyroptosis was also examined to identify its potential role in AP.

\section{Materials and methods}

Experimental rat model. In total, 24 Sprague-Dawley adult rats (male; age, 8-10 weeks; weight, 200-220 g) were purchased from the Experimental Animal Center of Guangxi Medical University. All rats were housed in normal barrier cages at a temperature of $25 \pm 2^{\circ} \mathrm{C}$ with $50-70 \%$ humidity using a 12 -h light/dark cycle and were allowed free access to food and water. All rats were checked every $6 \mathrm{~h}$. All animal experiments were conducted in accordance with the Institutional Animal Care and Use Committee of Guangxi Medical University (Nanning, China; approval no. 201910023). The rats were randomly divided into three groups: i) The control group; ii) the CAE-induced AP for $24 \mathrm{~h}(\mathrm{AP} 24 \mathrm{H})$ group; and iii) the CAE-induced AP for $48 \mathrm{~h}(\mathrm{AP} 48 \mathrm{H})$ group. The rats in the $\mathrm{AP} 24 \mathrm{H}$ and $\mathrm{AP} 48 \mathrm{H}$ groups were induced by intraperitoneal injections of CAE (Sigma-Aldrich; Merck KGaA). A total of seven injections at a dosage of $50 \mu \mathrm{g} / \mathrm{kg}$ CAE were administered once per hour. Animals were sacrificed 24 or $48 \mathrm{~h}$ after the last injection. The control group was similarly treated with the same volume of saline.

Cell culture. The human pancreatic duct epithelial HPDE6C7 cell line was purchased from Guangzhou Jennio Biotech Co., Ltd. HPDE6C7 cells were cultured in RPMI-1640 medium (Gibco; Thermo Fisher Scientific, Inc.) supplemented with $10 \% \mathrm{FBS}$ (Gibco) at $37^{\circ} \mathrm{C}$ in a $5 \% \mathrm{CO}_{2}$ incubator. The cells were divided into four groups according to different time points of stimulation with CAE: i Control; ii) treatment for $12 \mathrm{~h}(12 \mathrm{H})$; iii) treatment for $24 \mathrm{~h}(24 \mathrm{H})$; and iv) treatment for $48 \mathrm{~h}(48 \mathrm{H})$. The optimal CAE intervention time of $48 \mathrm{~h}$ was selected for subsequent experiments. Subsequently, HPDE6C7 cells were once again divided into four groups: i) The TRAF6 group; ii) the blank group; iii) the TRAF6 + CAE group; and iv) the blank + CAE group. TRAF6 was overexpressed by lentiviral infection in the TRAF6 group. The blank group was used as the negative control by transfecting with blank virus vector. Subsequently, the TRAF6 group and the blank group were stimulated CAE for $48 \mathrm{~h}$ as the TRAF6 + CAE group and blank + CAE group.

Lentivirus transfection. In order to facilitate the TRAF6 expression analysis, the second generation lentiviral packaging system was used. The study adopted the Ubi-MCS-3FLAG-CBh-gcGFP-IRES-puromycin lentivirus expression vector (Shanghai Genechem Co., Ltd.), and 293T cells (ATCC) were transformed with $32 \mu \mathrm{g}$ packaged plasmids [maintained in DMEM (Gibco; Thermo Fisher Scientific, Inc.) containing $10 \% \mathrm{FBS}$ at $37^{\circ} \mathrm{C}$ in a $5 \% \mathrm{CO}_{2}$ incubator]. The ratio of packaging vector:envelope plasmid was 1:1. The supernatant was collected 48 and $72 \mathrm{~h}$ after transfection, respectively. Next, the supernatant was filtered into an ultracentrifuge tube using a $0.45-\mu \mathrm{m}$ filter membrane. Centrifugation was performed at $72,000 \mathrm{xg}$ for $2 \mathrm{~h}$ at $4^{\circ} \mathrm{C}$. The supernatants were then discarded and the lentivirus deposition was resuspended with $500 \mu 1$ fresh medium and kept at $-80^{\circ} \mathrm{C}$. After 10 days, HPDE6C7 cells were infected with TRAF6 lentivirus vector using 0.1\% Polybrenne and Enhanced Infection Solution (Shanghai Genechem Co., Ltd.), with a multiplicity of infection of 5 at $37^{\circ} \mathrm{C}$. At $72 \mathrm{~h}$ post-infection, the medium was changed to fresh medium with $0.8 \mu \mathrm{g} / \mathrm{ml}$ puromycin. The uninfected wild-type cells were set as the control group, with an equal volume and concentration of puromycin. Fresh puromycin-containing medium was used as replacement medium every 3 or 4 days until the control group cells died. The stable cell lines were then cultured with $0.4 \mu \mathrm{g} / \mathrm{ml}$ puromycin. Reverse transcription-quantitative PCR and western blotting analyses were performed at $24 \mathrm{~h}$ post-transduction.

Blood and tissue preparation. All rats were anesthetized using $2 \%$ sodium pentobarbital $(45 \mathrm{mg} / \mathrm{kg})$ intraperitoneally. The abdominal cavity was opened and $2-3 \mathrm{ml}$ blood was then collected from the abdominal aorta. Subsequently, all rats were euthanized by rapid cervical dislocation and checked closely to confirm respiratory arrest. The pancreatic tissues around the pancreatic duct were removed. The blood samples were centrifuged for $10 \mathrm{~min}$ at $3,500 \mathrm{x}$ g at $4^{\circ} \mathrm{C}$ and serum samples were collected. Serum and pancreatic tissues were stored at $-80^{\circ} \mathrm{C}$ for further experiments.

Histopathological analysis. Fresh pancreatic tissues were fixed in $4 \%$ formaldehyde for $48 \mathrm{~h}$ at room temperature, followed by paraffin-embedding, sectioning and cutting into $4 \mu \mathrm{m}$ sections. The sections were deparaffinized and stained with H\&E (Beyotime institute of Biotechnology) for $8 \mathrm{~min}$ at room temperature. Images were captured using a light microscope (Olympus corporation). According to Van Laethem criteria (19), pancreatic injury was scored in terms of edema, inflammatory cell infiltration and acinar necrosis.

Serum and supernatant assays. Amylase (AMY) activity was determined using an automated clinical chemistry analyzer (Hitachi 7600; Hitachi, Ltd.). Serum levels of interleukin-1 $\beta$ (IL-1 $\beta$ ) (ELISA kit cat. no. ml037361; Shanghai Enzyme-linked Biotechnology Co., Ltd.) and IL-18 (ELISA kit cat. no. ml002816; Shanghai Enzyme-linked Biotechnology Co., Ltd.), and cell supernatants of IL-1 $\beta$ (ELISA kit cat. no. CSB-E08053h; Cusabio Technology, LLC) were analyzed according to the manufacturer's protocol.

Reverse transcription-quantitative PCR (RT-qPCR). Total RNA was extracted from the pancreatic tissues and HPDE6C7 cells using TRIzol ${ }^{\circledR}$ reagent (Invitrogen; Thermo Fisher Scientific, Inc.). RNA concentration and purity were determined using an ultra-micro spectrophotometer. Total RNA was reverse-transcribed into cDNA using a PrimeScript ${ }^{\mathrm{TM}}$ RT reagent kit with gDNA Eraser (cat. no. RR047A; Takara 
Biotechnology Co., Ltd.) according to the manufacturer's protocol. The Applied Biosystems 7500 qPCR system (Life Technologies Inc.) with SYBR-Green fluorescence was subsequently used for the qPCR. Primer sequences are listed in Table I. The following thermocycling conditions were used to amplify the cDNA in 40 cycles: Pre-denaturation $\left(95^{\circ} \mathrm{C}\right.$ for $30 \mathrm{sec})$, annealing $\left(95^{\circ} \mathrm{C}\right.$ for $\left.5 \mathrm{sec}\right)$ and extension $\left(60^{\circ} \mathrm{C}\right.$ for $20 \mathrm{sec}$ ). mRNA expression levels were calculated using the $2^{-\Delta \Delta \mathrm{Cq}}$ method (20) and normalized to the internal reference genes GAPDH or $\beta$-actin.

Western blotting. Total protein was extracted from pancreatic tissue and HPDE6C7 cells using a RIPA buffer with $1 \%$ phenylmethylsulfonyl fluoride. Total protein concentration was quantified using the Bicinchoninic Acid Protein Assay kit (Wuhan Boster Biological Technology Co., Ltd.). Protein samples $(30 \mu \mathrm{g} /$ well) were transferred onto polyvinylidene fluoride membranes following $10 \%$ and 15\% SDS-PAGE. Membranes were blocked with 5\% skimmed milk for 45 min at room temperature. The membranes were subsequently incubated with primary antibodies, including those for TRAF6 (1:2,000; cat. no. ab33915; Abcam), NLRP3 (1:1,000; cat. no. IMG-6668A; Novus Biologicals, Inc.), caspase-1 (1:1,000; cat. no. ab179515; Abcam), caspase-3 (1:1,000; cat. no. ab14220; Cell Signaling Technology, Inc.) and $\beta$-actin (1:5,000; cat. no. ab6276; Abcam) overnight at $4^{\circ} \mathrm{C}$. After washing with TBS-0.1\% Tween-20 buffer, membranes were incubated with goat anti-rabbit $\operatorname{IgG}$ (1:10,000; cat. no. ab4413; Cell Signaling Technology, Inc.) at room temperature for $1 \mathrm{~h}$. Blots were scanned using the Odyssey ${ }^{\circledR}$ Fc Imager system (LI-COR Biosciences), and protein expression was semi-quantified using imageJ software (version 1.4.1; National Institutes of Health) with $\beta$-actin as the loading control.

Hoechst/PI staining. HPDE6C7 cells were cultured with CAE for $48 \mathrm{~h}$ at $37^{\circ} \mathrm{C}$ and washed with PBS three times. Subsequently, $1 \mathrm{ml}$ cell staining buffer was added, followed by $5 \mu \mathrm{l}$ Hoechst staining solution and $5 \mu \mathrm{l}$ propidium iodide (PI) staining solution (Beyotime Institute of Biotechnology) at the same time and placed at $4^{\circ} \mathrm{C}$ for $30 \mathrm{~min}$ in the dark. After washing with PBS three times, the dye mixture was discarded and the cells were observed under a fluorescence microscope.

Statistical analysis. All data are presented as the mean \pm standard of at least three independent experiments. Data were analyzed using SPSS 24.0 software (IBM Corp.) and are expressed as the mean \pm standard deviation. One-way ANOVA was used for statistical comparisons between more than two groups, followed by Tukey's post hoc test. $\mathrm{P}<0.05$ was considered to indicate a statistically significant difference.

\section{Results}

Pancreatic histopathological scores. Pancreatic injury was evaluated using a histopathological scoring system. Histological changes confirmed that AP was successfully established in the rats. Edema and inflammatory cell infiltration were observed in the pancreatic tissues of AP rats, and acinar necrosis was occasionally observed in the AP48H group (Fig. 1A). The pancreatic pathological scores of the AP24H
Table I. Sequences of primers used for reverse transcription-quantitative PCR.

\begin{tabular}{ll}
\hline Gene & \multicolumn{1}{c}{ Sequence (5'-3') } \\
\hline Human- & F: CGCGCATAGAACGACAAG \\
TRAF6 & R: TTTCCAGGGGTGGGTCAAAC \\
Human- & F: CGGCAAGACCAAGACGTGTGAG \\
NLRP3 & R: CAGGCTCAGAATGCTCATCATCGG \\
Human- & F: GAAGAAACACTCTGAGCAAGTC \\
caspase-1 & R: GATGATGATCACCTTCGGTTTG \\
Human- & F: AGATGTCCAGCCAGCTGCACC \\
caspase-3 & R: TGACCCCACCGAACTCAAAGA \\
Human- & F: CAAATTCCATGGCACCGTCA \\
GAPDH & R: GACTCCACGACGTACTCAGC \\
Rat-TRAF6 & F: TTTGGCGTCGGAGACACTTG \\
Rat-NLRP3 & R: TCGCTTGAAGACTGGCTGGA \\
& R: CTGAAGCATCTGCTCTGCAACC \\
Rat-caspase-1 & F: ACTCGTACACGTCTTGCCCTCA \\
& R: CTGGGCAGGCAGCAAATTC \\
Rat-caspase-3 & F:AGACAGACAGTGGAACTGACGATG \\
Rat- 3 -actin & R: GGCGCAAAGTGACTGGATGA \\
& F: TTGCTGACAGGATGCAGAA \\
& R: ACCAATCCACACAGAGTACTT \\
\hline
\end{tabular}

TRAF6, tumor necrosis factor receptor-associated factor 6; NLRP3, NLR pyrin domain containing 3; F, forward; R, reverse.

group were significantly higher compared with the control group. Compared with the AP24H group, the AP48H group displayed significantly increased pancreatic injury (Fig. 1B).

AMY, IL- $1 \beta$ and $I L-18$ serum levels. AMY activity and serum levels of IL-1 $\beta$ and IL-18 were detected. The serum activity of AMY was markedly higher in the AP24H group compared with that in the control group (Fig. 1C). IL-1 $\beta$ and IL-18 serum levels in the AP groups were significantly higher compared with those in the control group. Furthermore, compared with the levels in the AP24H group, the levels in the AP48H group increased to a greater extent (Fig. 1D and E).

mRNA and protein expression levels of TRAF6, NLPR3, caspase-1 and caspase-3 in pancreatic tissues. Following induction with CAE for 24 and $48 \mathrm{~h}$ in rats, TRAF6 and caspase-1/3 pathways were examined by RT-qPCR and western blotting. The mRNA and protein expression levels of TRAF6, NLPR3, caspase-1 and caspase-3 were significantly upregulated in the pancreatic tissues of the AP groups compared with the control group. Moreover, the AP48H group demonstrated significantly higher mRNA and protein expression levels compared with the AP24H group (Fig. 2A-I).

Construction of lentivirus transfection vector for overexpression of TRAF6. In order to investigate the potential role of TRAF6 in vitro, TRAF6 was overexpressed in HPDE6C7 cells using lentiviral infection, and the transfection data are described in our previous study (21). 


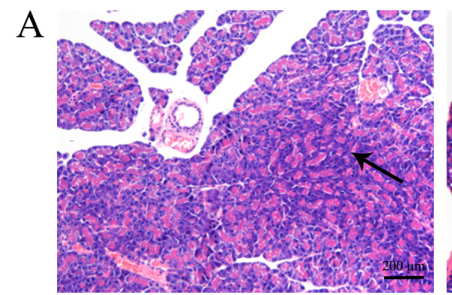

Control

B

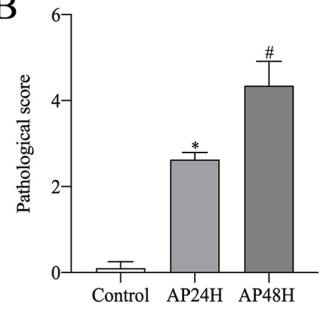

D

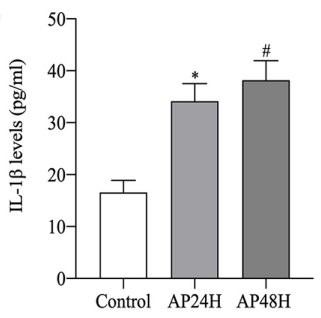

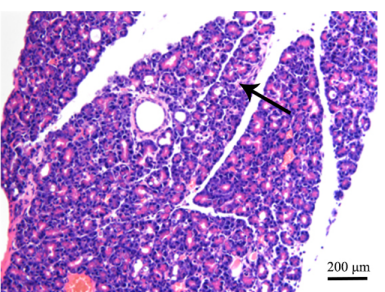

AP24H

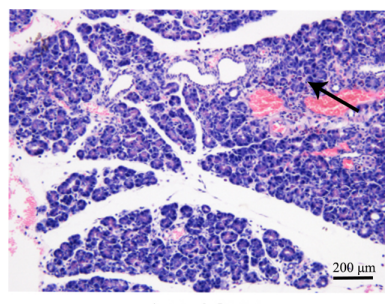

AP48H
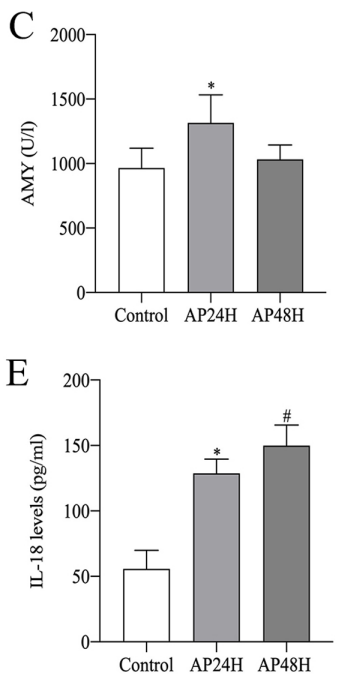

Figure 1. Histological changes in the pancreas, serum AMY activity, and IL-1 $\beta$ and IL-18 levels. (A) Histopathological changes (arrows) in H\&E-stained pancreatic tissues (magnification, $x 200$ ). (B) Pathological scores of the pancreas. (C) Serum AMY activity. Serum (D) IL-1 $\beta$ and (E) IL-18 levels in the different groups. "P<0.05 vs. control; " $\mathrm{P}<0.05$ vs. control and AP24H. AMY, amylase; AP24H, caerulein-induced acute pancreatitis for 24 h; AP48H, caerulein-induced acute pancreatitis for $48 \mathrm{~h}$.

Pyroptosis morphology of HPDE6C7 cells following CAE treatment and TRAF6 overexpression. HPDE6C7 cells were treated with CAE and TRAF6 overexpression vectors and visualized using Hoechst/PI staining. Compared with the control, in the $12 \mathrm{H}$ group, PI could pass through the cell membrane into the nucleus and displayed red fluorescence following treatment with CAE. Red fluorescence was markedly increased in the $24 \mathrm{H}$ and $48 \mathrm{H}$ groups compared with the control and 12H group (Fig. 3A). Following TRAF6 overexpression, a small amount of red fluorescence was observed in the TRAF6 group, compared with the blank group where none was observed. After CAE treatment, the red fluorescence increased in both groups, and that in the TRAF6 + CAE group was significantly higher than that in the blank + CAE group (Fig. 3B).

$I L-1 \beta$ levels in the supernatant of HPDE6C7 cells. The level of IL-1 $\beta$ was also detected in the HPDE6C7 cell supernatant. IL-1 $\beta$ levels in CAE-stimulated groups were significantly higher compared with the control group. Furthermore, IL-1 $\beta$ levels increased with CAE stimulation time, with the highest IL-1 $\beta$ level observed in the $48 \mathrm{H}$ group (Fig. 4A). Moreover, IL-1 $\beta$ levels in the cell supernatants of the TRAF6 group and the TRAF6 + CAE group were markedly higher compared with the blank group and the blank + CAE group, respectively (Fig. 4B).

mRNA and protein expression levels of NLPR 3, caspase-1 and caspase-3 in HPDE6C7 cells. As the potential mechanisms of the caspase-1/3 signaling pathways were investigated in rats, this process was also explored in vitro. NLPR 3, caspase-1 and caspase-3 mRNA and protein expression levels were analyzed via RT-qPCR and western blotting in HPDE6C7 cells. mRNA expression levels of NLRP3 and caspase-1 significantly increased with CAE stimulation compared with the control. NLRP3 mRNA expression increased in a time-dependent manner when exposed to CAE, with the highest expression levels observed in the $48 \mathrm{H}$ group (Fig. 4C). Caspase-1 mRNA expression levels were highest in the $12 \mathrm{H}$ group, with all CAE-stimulated groups being significantly higher compared with the control (Fig. 4D).

NLRP3 and caspase-1 mRNA and cleaved caspase-1 protein expression levels in the TRAF6 group were significantly lower compared with the blank group (Fig. 5A, B and F), whereas the protein expression levels of NLRP3 in the TRAF6 group were significantly higher compared with the blank group (Fig. 5D and E). Caspase-3 mRNA and cleaved caspase-3 protein expression levels in the TRAF6 group were significantly higher compared with that in the blank group (Fig. 5C, D and G). Following TRAF6 overexpression, the mRNA expression levels of NLRP3 and caspase-3 were significantly increased in the TRAF6 + CAE group compared with the blank + CAE group (Fig. 5A and C). However, caspase-1 mRNA expression was significantly decreased in the TRAF6 + CAE group compared with the blank + CAE group (Fig. 5B). Protein expression levels of NLRP3, cleaved caspase- 1 and cleaved caspase- 3 in the TRAF6 + CAE group were significantly higher compared with the blank + CAE group (Fig. 5D-G). 
A

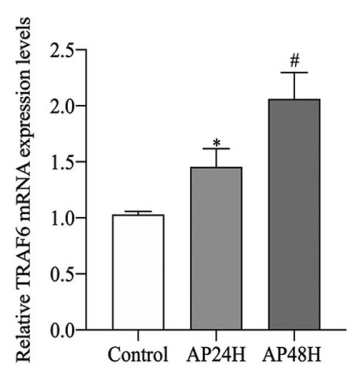

D

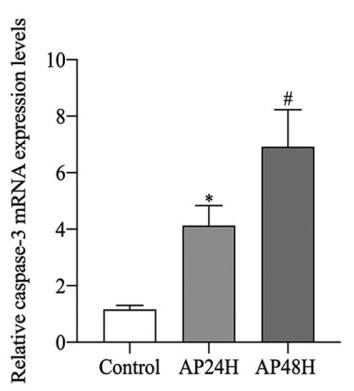

$\mathrm{G}$

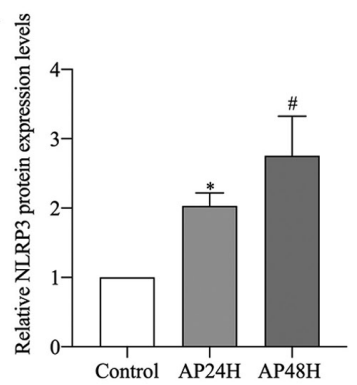

$\mathrm{B}$

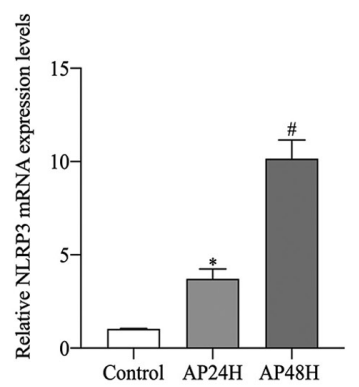

E

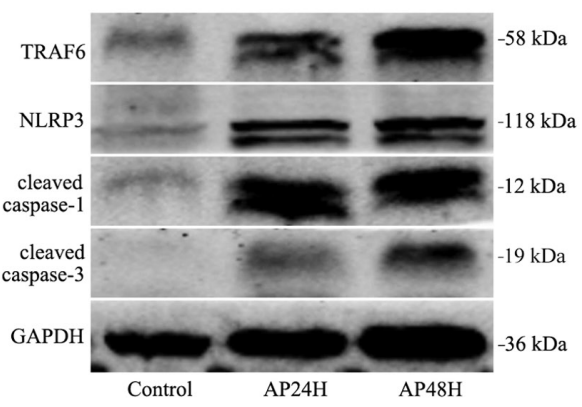

H

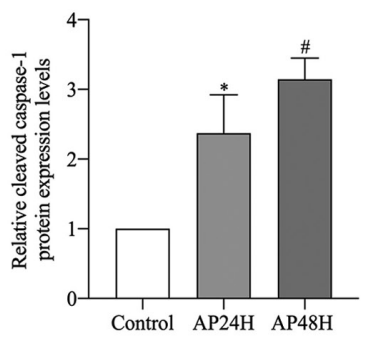

C

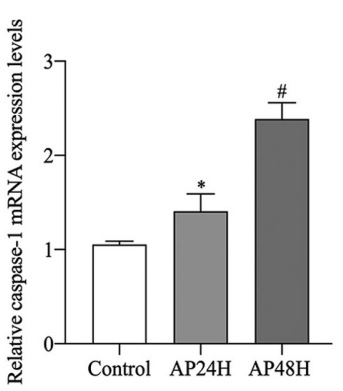

F

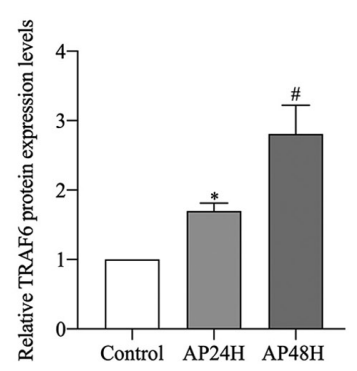

I

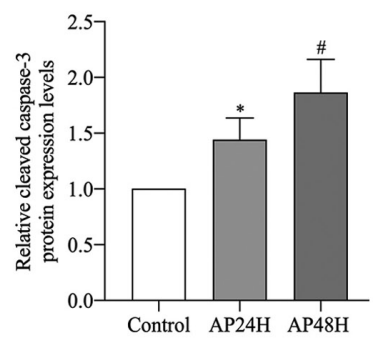

Figure 2. TRAF6, NLPR3, caspase-1 and caspase-3 mRNA and protein expression levels in pancreatic tissue. Reverse transcription-quantitative PCR analysis was performed to determine the expression levels of (A) TRAF6, (B) NLRP3, (C) caspase-1 and (D) caspase-3. (E) Western blotting was performed to determine the protein expression levels of (F) TRAF6 (58 kDa), (G) NLRP3 (118 kDa), (H) cleaved caspase-1 (12 kDa) and (I) cleaved caspase-3 (19 kDa). ${ }^{*} \mathrm{P}<0.05$ vs. control; " $\mathrm{P}<0.05$ vs. control and AP24H. TRAF6, tumor necrosis factor receptor-associated factor 6; NLRP3, NLR pyrin domain containing 3; AP24H, caerulein-induced acute pancreatitis for $24 \mathrm{~h}$; AP48H, caerulein-induced acute pancreatitis for $48 \mathrm{~h}$.

\section{Discussion}

AP is a common inflammatory disease that is often characterized by abnormal activation of trypsin in the pancreas, resulting in severe local inflammatory reactions, including edema and bleeding and necrosis of pancreatic tissues (22). Further disease progression can be accompanied by heart, lung, kidney and intestinal injury as well as other complications, thereby increasing the risk of death (23-25).

Pyroptosis is morphologically characterized by both necrosis and apoptosis. During pyroptosis, the swelling of cells results in the formation of vesicular projections and numerous 1-2 nm pores on the cell membrane (26). With the rupture of the cell membrane, cytoplasmic contents are released into the extracellular space, which induces an inflammatory reaction, changes nuclear concentration and causes DNA rupture, as well as other changes (27). Pyroptosis activation pathways are divided into classical caspase-1-dependent signaling pathways and non-classical caspase-4/5/11-dependent signaling pathways, although a previous study has shown that caspase- 3 may also be involved in pyroptosis (28).
NLRs are known to serve a significant role in pyroptosis. The NLRP3 inflammasome consists of NLRP3 protein, ASC and pro-caspase-1 (29). Pathogen-associated molecular patterns, such as bacteria and viruses, or damage-associated molecular patterns (DAMPs), are recognized by intracellular and extracellular pattern recognition receptors, which activate NLRP3 inflammasome assembly, after which the NLRP3 inflammasome cleaves pro-caspase-1 into active caspase-1 (30). Furthermore, activated caspase-1 converts pro-IL-1 $\beta$ and pro-IL-18 to mature IL-1 $\beta$ and IL-18, respectively. Therefore, pyroptosis results in the release of IL-1 $\beta$ and IL-18, which recruit inflammatory cells and amplify the inflammatory response (8). NLRP3-deficient mice display a greater reduction in the inflammatory response of the AP model, suggesting that NLRP3 may serve a pivotal role in AP (31). A recent study suggested that NLRP3 induces the T helper 2 cell-mediated response via IL-18, thus facilitating the development of the inflammatory response in mice with AP and inhibition of NLRP3 may be useful to treat patients with severe AP (32). The present study demonstrated that AP contributed to a significant increase in the mRNA and protein 


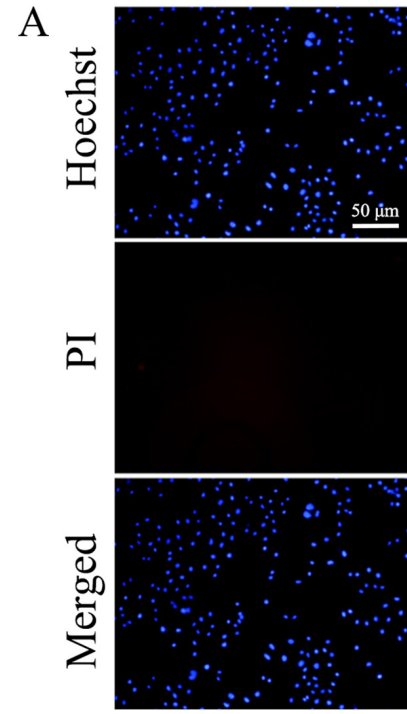

Control

B

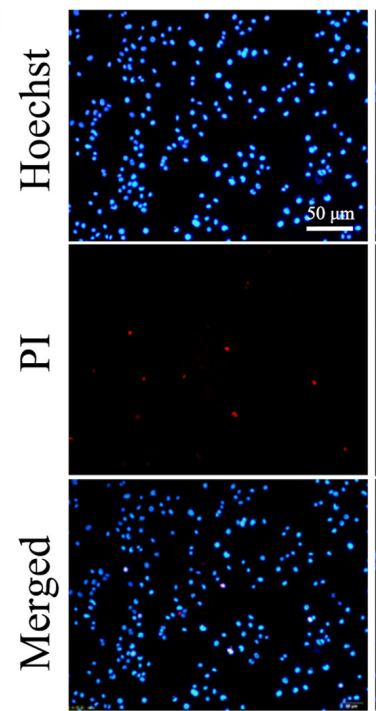

TRAF6

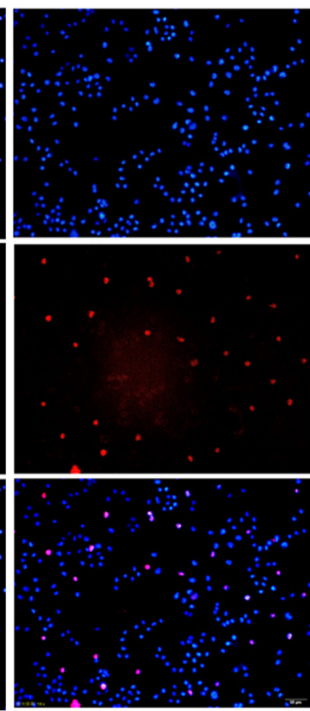

$12 \mathrm{H}$

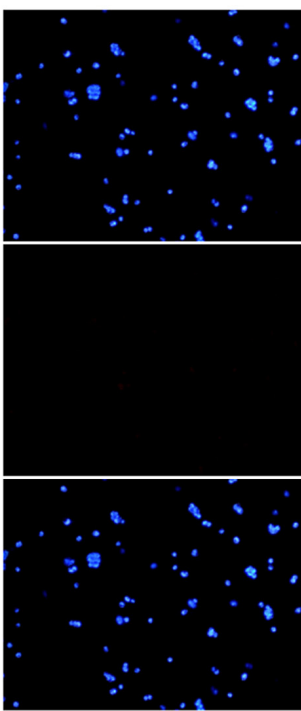

Blank

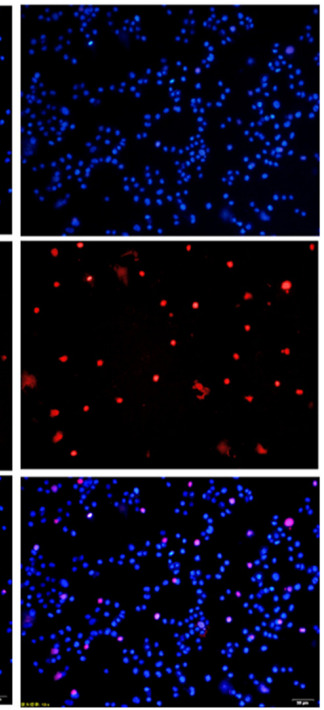

$24 \mathrm{H}$

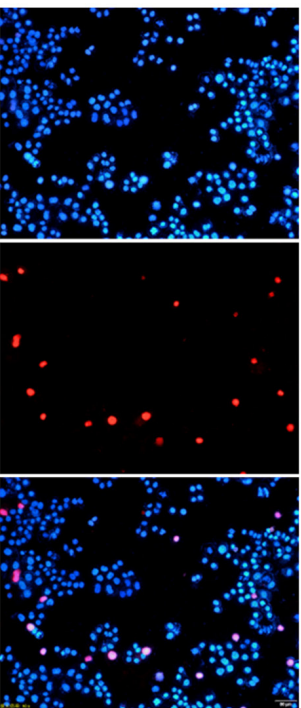

TRAF6 + CAE

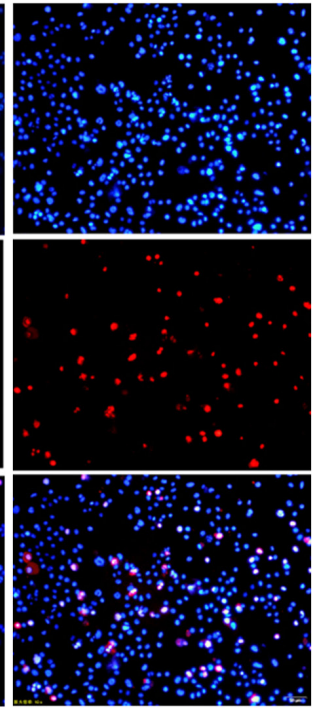

$48 \mathrm{H}$

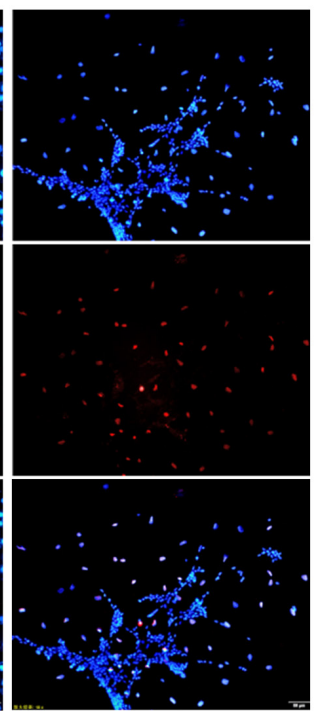

Blank + CAE

Figure 3. Pyroptosis in HPDE6C7 cells as observed by Hoechst/PI staining (magnification, x50). (A) HPDE6C7 cells were treated with CAE at 12, 24 and 48 h. (B) Following TRAF6 overexpression, HPDE6C7 cells were treated with CAE. 12H, CAE treatment for 12 h; $24 \mathrm{H}$, CAE treatment for 24 h; 48H, CAE treatment for $48 \mathrm{~h}$; CAE, caerulein; TRAF6, tumor necrosis factor receptor-associated factor 6.

expression levels of NLRP3, caspase-1 and caspase-3, and the serum levels of IL-1 $\beta$ and IL-18. These results indicated that NLRP3, caspase-1 and caspase-3 may serve a role in CAE-induced AP in rats.

TLR is a crucial component of the innate immune system, which is involved in the occurrence of AP through the activation of the NLRP3 inflammasome (33). TLR9 is an important upstream DAMP receptor that is involved in the pathogenesis of experimentally induced pancreatitis, along with NLRP3, ASC and caspase-1, and more importantly, that NLRP3, ASC and caspase-1 are required for inflammation in AP (34), with TRAF6 being the central confluence point and key adaptor protein of most TLR signaling pathways. TRAF6 is a ubiquitin ligase that mediates the occurrence of acute and chronic inflammatory diseases (35). A previous study demonstrated that TRAF6 is a convergence point of the TLR4 signaling pathway, which is critical in AP (36). Hence, in the present study TRAF6 expression levels were detected in the pancreatic tissues. The results indicated that TRAF6 expression was significantly increased in CAE-induced AP.

In the present study, the inflammatory response and pancreatic injury worsened within 24 and $48 \mathrm{~h}$ following AP induction in rats. Serum levels of AMY, IL-1 $\beta$ and IL-18 were increased. Simultaneously, the mRNA and protein expression levels of TRAF6, NLRP3, caspase-1 and caspase-3 were also significantly elevated in pancreatic tissues. These results indicated that the TRAF6, NLRP3 and caspase-1/3 signaling pathways, are also involved in the pathogenesis of AP. It was therefore hypothesized that pancreatic tissue injury may be associated with the activation of pyroptosis, which leads to an inflammatory response.

To investigate the potential mechanisms by which TRAF6 is involved in pyroptosis, HPDE6C7 cells were treated with CAE to induce and observe pyroptosis. Hoechst/PI staining 
A

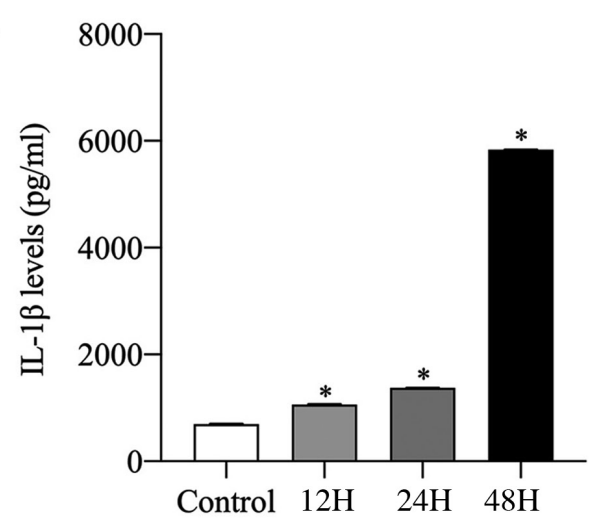

$\mathrm{C}$

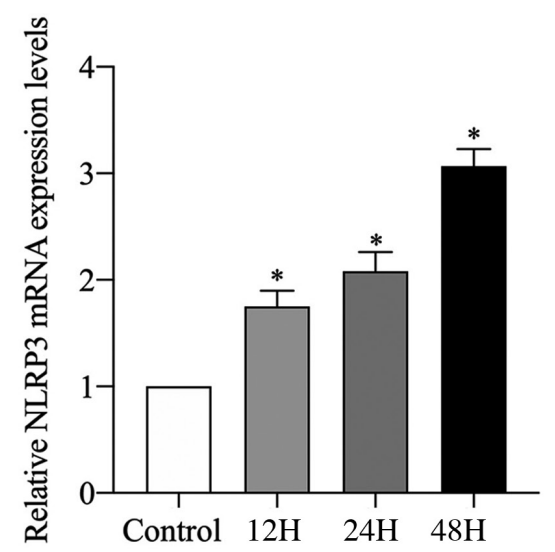

B

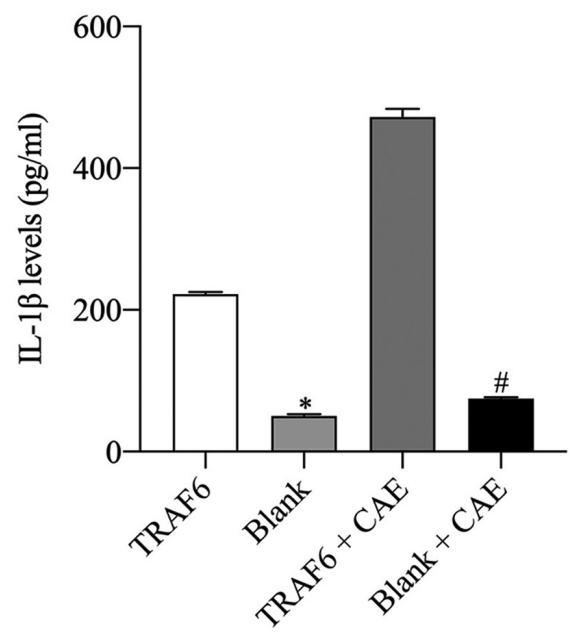

$\mathrm{D}$

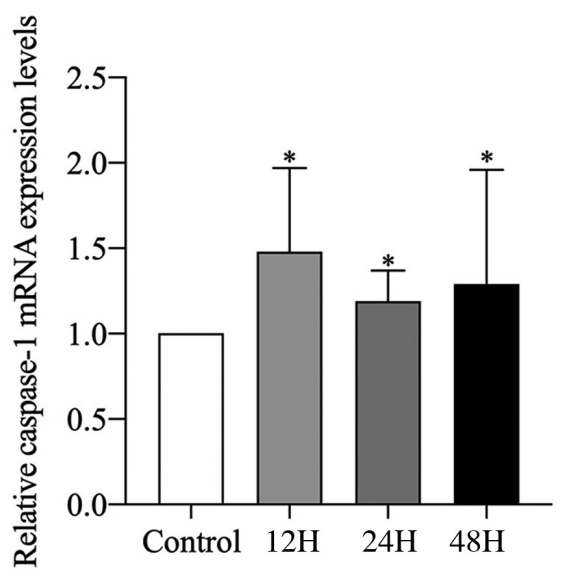

Figure 4. IL-1 $\beta$ levels and NLRP3 and caspase-1 mRNA expression levels in HPDE6C7 cells. IL-1 $\beta$ levels in HPDE6C7 cells (A) after CAE stimulation for 12, 24 and $48 \mathrm{~h}$ and (B) following TRAF6 overexpression and CAE stimulation. Reverse transcription-quantitative PCR analysis of (C) NLRP3 and (D) caspase-1 in HPDE6C7 cells following CAE stimulation for 12,24 and $48 \mathrm{~h}$. ${ }^{*} \mathrm{P}<0.05$ vs. control or TRAF6; ${ }^{\text {\# }}<0.05$ vs. TRAF6 + CAE. TRAF6, tumor necrosis factor receptor-associated factor 6; NLRP3, NLR pyrin domain containing 3; 12H, CAE treatment for $12 \mathrm{~h}$; $24 \mathrm{H}$, CAE treatment for $24 \mathrm{~h}$; $48 \mathrm{H}$, CAE treatment for 48 h; CAE, caerulein.

demonstrated that following treatment with CAE, extensive red fluorescence appeared in HPDE6C7 cells at 12, 24 and $48 \mathrm{~h}$, especially at $48 \mathrm{~h}$. PI staining can also demonstrate the rounding, swelling and necrosis of cells. Since the integrity of the cell membrane is destroyed during necrosis, PI can freely pass through the cell membrane and as a result cells exhibit red fluorescence, which is considered to represent pyroptosis $(37,38)$. Following TRAF6 overexpression, a small amount of necrosis was found in the TRAF6 group, while CAE-stimulated cells for $48 \mathrm{~h}$ exhibited higher red fluorescence in the TRAF6 + CAE group and the Blank + CAE group.

The present study also confirmed that supernatant IL-1 $\beta$, NLRP3 and caspase-1 mRNA expression levels significantly increased following CAE treatment of HPDE6C7 cells. However, TRAF6 overexpression significantly decreased NLRP3 mRNA expression in the TRAF6 group compared with the blank group. The detection of the mRNA expression levels of numerous genes cannot predict their protein expression levels (39). Since the effector molecule of a gene is the protein and not the RNA, NLRP3 protein expression was analyzed via western blotting. Western blotting demonstrated that NLRP3 protein expression levels in the TRAF6 group were significantly higher compared with the blank group. Furthermore, cleaved caspase-1 protein expression levels were significantly decreased in the TRAF6 group compared with the blank group. However, cleaved caspase-1 protein expression levels were significantly higher in the TRAF6 + CAE group than in the blank + CAE group following CAE treatment. This result indicated that TRAF6 overexpression may lead to a decrease in cleaved caspase-1 protein expression levels under viral transduction and an increase in cleaved caspase-1 when treated with CAE in the TRAF6 + CAE group. Significantly increased supernatant IL-1 $\beta$, caspase-3 mRNA and protein expression levels were also observed in the TRAF6 and TRAF6 + CAE groups. The results of the present study therefore indicated that CAE treatment and TRAF6 overexpression increased the aforementioned parameters and therefore indicated the activation of pyroptosis.

In conclusion, TRAF6 and the caspase-1/3 signaling pathways served a role in CAE-induced AP in rats. Pyroptosis in AP was attributed to a combination of CAE and TRAF6 in the human pancreatic ductal epithelial cells. In this study, it was preliminarily found that TRAF6 may be related to the pyroptosis of AP. These findings provided evidence to support further investigations into TRAF6 in pyroptosis during AP. 

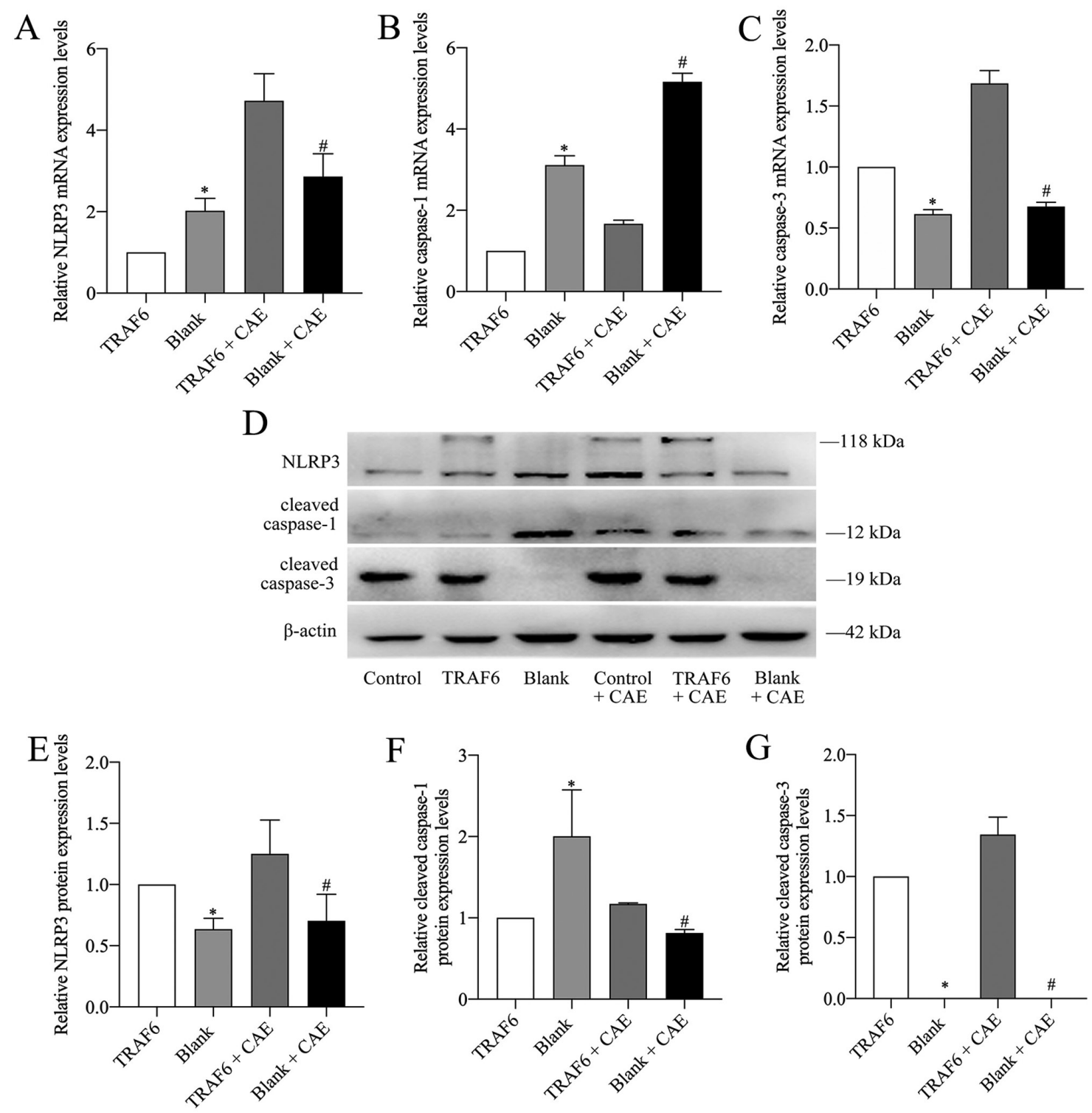

Figure 5. NLPR3, cleaved caspase-1 and cleaved caspase-3 mRNA and protein expression levels in HPDE6C7 cells. Following TRAF6 overexpression, reverse transcription-quantitative PCR analysis was performed to determine the mRNA expression levels of (A) NLRP3, (B) caspase-1 and (C) caspase-3. (D) Western blotting was performed to determine the protein expression levels of (E) NLRP3 (118 kDa), (F) cleaved caspase-1 (12 kDa) and (G) cleaved caspase-3 (19 kDa). ${ }^{*} \mathrm{P}<0.05$ vs. TRAF6; ${ }^{\#} \mathrm{P}<0.05$ vs. TRAF6 + CAE. Control, HPDE6C7 cell; TRAF6, tumor necrosis factor receptor-associated factor 6; NLRP3, NLR pyrin domain containing 3 ; CAE, caerulein.

\section{Acknowledgements}

Not applicable.

\section{Funding}

The present study was supported by the National Natural Science Foundation of China (grant no. 81960126), the Nanning Qingxiu District Science and Technology Project (grant no. 2019026) and the Guangxi Health Committee Self-Funded Research Project (grant no. Z20190894).

\section{Availability of data and materials}

The datasets used and/or analyzed during the current study are available from the corresponding author on reasonable request.

\section{Authors' contributions}

BW, YG and ZL contributed to the design, analysis and interpretation of the study. BW, YG, HY and JZ contributed to the data collection, data analysis and preparation of the manuscript. ZS contributed materials and assisted in the data analysis. BW, YG and ZS contributed to the writing and revision of the manuscript. BW and YG confirm the authenticity of all the raw data. All authors have read and approved the final manuscript.

\section{Ethics approval and consent to participate}

The present study was approved by the Institutional Animal Care and Use Committee of Guangxi Medical University (Nanning, China; approval no. 201910023). 


\section{Patient consent for publication}

Not applicable.

\section{Competing interests}

The authors declare that they have no competing interests.

\section{References}

1. Shah AP, Mourad MM and Bramhall SR: Acute pancreatitis: Current perspectives on diagnosis and management. J Inflamm Res 11: 77-85, 2018.

2. Sendler M, Mayerle J and Lerch MM: Necrosis, apoptosis, necroptosis, pyroptosis: It matters how acinar cells die during pancreatitis. Cell Mol Gastroenterol Hepatol 2: 407-408, 2016.

3. Wang Q, Liu S and Han Z: miR-339-3p regulated acute pancreatitis induced by caerulein through targeting TNF receptor-associated factor 3 in AR42J cells. Open Life Sci 15: 912-922, 2020.

4. Wu K, Yao G, Shi X, Zhang H, Zhu Q, Liu X, Lu G, Hu L, Gong W, Yang Q, et al: Asiaticoside ameliorates acinar cell necrosis in acute pancreatitis via toll-like receptor 4 pathway. Mol Immunol 130: 122-132, 2021.

5. Song G, Ma Z, Liu D, Qian D, Zhou J, Meng H, Zhou B and Song Z: Bone marrow-derived mesenchymal stem cells attenuate severe acute pancreatitis via regulation of microRNA-9 to inhibit necroptosis in rats. Life Sci 223: 9-21, 2019.

6. Lin T, Song J, Pan X, Wan Y, Wu Z, Lv S, Mi L, Wang Y and Tian F: Downregulating gasdermin D reduces severe acute pancreatitis associated with pyroptosis. Med Sci Monit 27: e927968, 2021.

7. Fischer FA, Chen KW and Bezbradica JS: Posttranslational and therapeutic control of gasdermin-mediated pyroptosis and inflammation. Front Immunol 12: 661162, 2021.

8. Man SM, Karki R and Kanneganti TD: Molecular mechanisms and functions of pyroptosis, inflammatory caspases and inflammasomes in infectious diseases. Immunol Rev 277: 61-75, 2017.

9. Jia C, Chen H, Zhang J, Zhou K, Zhuge Y, Niu C, Qiu J, Rong X, Shi Z, Xiao J, et al: Role of pyroptosis in cardiovascular diseases. Int Immunopharmacol 67: 311-318, 2019.

10. Teng JF, Mei QB, Zhou XG, Tang Y, Xiong R, Qiu WQ, Pan R, Law BY, Wong VK, Yu CL, et al: Polyphyllin VI induces caspase 1 mediated pyroptosis via the induction of ROS/NF $\kappa \mathrm{B} / \mathrm{NLRP} 3 / \mathrm{GSDMD}$ signal axis in non small cell lung cancer. Cancers (Basel) 12: 193, 2020.

11. Wu C, Chen H, Zhuang R, Zhang H, Wang Y,Hu X, Xu Y, Li J,Li Y, Wang $\mathrm{X}$, et al: Betulinic acid inhibits pyroptosis in spinal cord injury by augmenting autophagy via the AMPK-mTOR-TFEB signaling pathway. Int J Biol Sci 17: 1138-1152, 2021.

12. Cheng $\mathrm{L}$ and Zhang W: DJ-1 affects oxidative stress and pyroptosis in hippocampal neurons of Alzheimer's disease mouse model by regulating the Nrf2 pathway. Exp Ther Med 21: $557,2021$.

13. Lin KM, Hu W, Troutman TD, Jennings M, Brewer T, Li X, Nanda S, Cohen P, Thomas JA and Pasare C: IRAK-1 bypasses priming and directly links TLRs to rapid NLRP3 inflammasome activation. Proc Natl Acad Sci USA 111: 775-780, 2014 Erratum in: Proc Natl Acad Sci USA 111: 3195, 2014.

14. Bortoluci KR and Medzhitov R: Control of infection by pyroptosis and autophagy: Role of TLR and NLR. Cell Mol Life Sci 67: 1643-1651,2010.

15. Ringel-Scaia VM, McDaniel DK and Allen IC: The goldilocks conundrum: NLR inflammasome modulation of gastrointestina inflammation during inflammatory bowel disease. Crit Rev Immunol 36: 283-314, 2016.

16. Walsh MC, Lee J and Choi Y: Tumor necrosis factor receptor-associated factor 6 (TRAF6) regulation of development, function, and homeostasis of the immune system. Immunol Rev 266: 72-92, 2015.

17. Yoon K, Jung EJ, Lee SR, Kim J, Choi Y and Lee SY: TRAF6 deficiency promotes TNF-induced cell death through inactivation of GSK3beta. Cell Death Differ 15: 730-738, 2008.

18. Xing Y, Yao X, Li H, Xue G, Guo Q, Yang G, An L, Zhang Y and Meng G: Cutting edge: TRAF6 mediates TLR/IL 1R signaling induced nontranscriptional priming of the NLRP3 inflammasome. J Immunol 199: 1561-1566, 2017.
19. Van Laethem JL, Marchant A, Delvaux A, Goldman M, Robberecht $\mathrm{P}$, Velu $\mathrm{T}$ and Devière $\mathrm{J}$ : Interleukin 10 prevents necrosis in murine experimental acute pancreatitis. Gastroenterology 108: 1917-1922, 1995.

20. Livak KJ and Schmittgen TD: Analysis of relative gene expression data using real-time quantitative PCR and the $2(-\Delta \Delta \mathrm{C}(\mathrm{T}))$ Method. Methods 25: 402-408, 2001.

21. Wei BW, Gong YH, Su Z, Yang HY, Qin MB and Liang ZH: Involvement of miR $125 \mathrm{~b}$ in human pancreatic ductal epithelial barrier affected by TRAF6. Basic Clin Med 41: 1272-1276, 2021. (In Chinese).

22. Yu JH and Kim H: Oxidative stress and inflammatory signaling in cerulein pancreatitis. World J Gastroenterol 20: 17324-17329, 2014.

23. Garg PK and Singh VP: Organ failure due to systemic injury in acute pancreatitis. Gastroenterology 156: 2008-2023, 2019.

24. Wang B, Li XH, Song Z, Li ML, Wu XW, Guo MX, Zhang XH and Zou XP: Isoacteoside attenuates acute kidney injury induced by severe acute pancreatitis. Mol Med Rep 23: 1-10, 2021.

25. Hu J, Zhang YM, Miao YF, Zhu L, Yi XL, Chen H, Yang XJ, Wan MH and Tang WF: Effects of Yue-Bi-Tang on water metabolism in severe acute pancreatitis rats with acute lung-kidney injury. World J Gastroenterol 26: 6810-6821, 2020.

26. Fink SL and Cookson BT: Caspase-1-dependent pore formation during pyroptosis leads to osmotic lysis of infected host macrophages. Cell Microbiol 8: 1812-1825, 2006.

27. Shi J, Zhao Y, Wang K, Shi X, Wang Y, Huang H, Zhuang Y, Cai T, Wang F and Shao F: Cleavage of GSDMD by inflammatory caspases determines pyroptotic cell death. Nature 526: 660-665, 2015

28. Wang Y, Gao W, Shi X, Ding J, Liu W, He H, Wang K and Shao F: Chemotherapy drugs induce pyroptosis through caspase-3 cleavage of a gasdermin. Nature 547: 99-103, 2017.

29. Sutterwala FS, Ogura Y, Zamboni DS, Roy CR and Flavell RA: NALP3: A key player in caspase-1 activation. J Endotoxin Res 12: 251-256, 2006.

30. Martins JD, Liberal J, Silva A, Ferreira I, Neves BM and Cruz MT: Autophagy and inflammasome interplay. DNA Cell Biol 34: 274-281, 2015.

31. Fu Q, Zhai Z, Wang Y, Xu L, Jia P, Xia P, Liu C, Zhang X, Qin T and Zhang H: NLRP3 deficiency alleviates severe acute pancreatitis and pancreatitis associated lung injury in a mouse model. BioMed Res Int 2018: 1294951, 2018

32. Sendler M, van den Brandt C, Glaubitz J, Wilden A, Golchert J, Weiss FU, Homuth G, De Freitas Chama LL, Mishra N, Mahajan UM, et al: NLRP3 inflammasome regulates development of systemic inflammatory response and compensatory anti inflammatory response syndromes in mice with acute pancreatitis. Gastroenterology 158: 253-269.e14, 2020.

33. Liu Y, Li Y, Chen KL, Zhou B, Lv ZY, Zhou ZG and Li Y: Knockdown of myeloid differentiation factor 88 attenuates lipopolysaccharide Induced inflammatory response in pancreatic ductal cells. Pancreas 45: 755-760, 2016.

34. Hoque R, Sohail M, Malik A, Sarwar S, Luo Y, Shah A, Barrat F, Flavell R, Gorelick F, Husain S, et al: TLR9 and the NLRP3 inflammasome link acinar cell death with inflammation in acute pancreatitis. Gastroenterology 141: 358-369, 2011.

35. Abdullah M, Berthiaume JM and Willis MS: Tumor necrosis factor receptor-associated factor 6 as a nuclear factor kappa B-modulating therapeutic target in cardiovascular diseases: At the heart of it all. Transl Res 195: 48-61, 2018.

36. Zhou XY, Zhou ZG, Ding JL, Wang L, Wang R, Zhou B, Gu J, Sun XF and Li Y: TRAF6 as the key adaptor of TLR4 signaling pathway is involved in acute pancreatitis. Pancreas 39: 359-366, 2010.

37. Brennan MA and Cookson BT: Salmonella induces macrophage death by caspase-1-dependent necrosis. Mol Microbiol 38: 31-40, 2000.

38. Yang Y, Liu PY, Bao W, Chen SJ, Wu FS and Zhu PY: Hydrogen inhibits endometrial cancer growth via a ROS/NLRP3/caspase-1/GSDMD-mediated pyroptotic pathway. BMC Cancer 20: 28, 2020.

39. Chen G, Gharib TG, Huang CC, Taylor JM, Misek DE, Kardia SL Giordano TJ, Iannettoni MD, Orringer MB, Hanash SM, et al: Discordant protein and mRNA expression in lung adenocarcinomas. Mol Cell Proteomics 1: 304-313, 2002.

This work is licensed under a Creative Commons Attribution-NonCommercial-NoDerivatives 4.0 International (CC BY-NC-ND 4.0) License. 